\title{
Decreased C-reactive protein induces abnormal vascular structure in a rat model of liver dysfunction induced by bile duct ligation
}

\author{
Ji Hye Jun', Jong Ho Choi', Si Hyun Bae' ${ }^{2}$ Seh Hoon $\mathrm{Oh}^{3}$, and Gi Jin Kim \\ 'Department of Biomedical Science, CHA University, Seongnam, ${ }^{2}$ Department of Internal Medicine, Catholic University Medical \\ College, Seoul, Korea; ${ }^{3}$ Department of Pathology, Immunology and Laboratory Medicine, University of Florida, College of Medicine, \\ Gainsville, FL, USA
}

Background/Aims: Chronic liver disease leads to liver fibrosis, and although the liver does have a certain regenerative capacity, this disease is associated with dysfunction of the liver vessels. C-reactive protein (CRP) is produced in the liver and circulated from there for metabolism. CRP was recently shown to inhibit angiogenesis by inducing endothelial cell dysfunction. The objective of this study was to determine the effect of CRP levels on angiogenesis in a rat model of liver dysfunction induced by bile duct ligation (BDL).

Methods: The diameter of the hepatic vein was analyzed in rat liver tissues using hematoxylin and eosin (H\&E) staining. The expression levels of angiogenic factors, albumin, and CRP were analyzed by real-time PCR and Western blotting. A tube formation assay was performed to confirm the effect of CRP on angiogenesis in human umbilical vein endothelial cells (HUVECs) treated with lithocholic acid (LCA) and siRNA-CRP.

Results: The diameter of the hepatic portal vein increased significantly with the progression of cirrhosis. The expression levels of angiogenic factors were increased in the cirrhotic liver. In contrast, the expression levels of albumin and CRP were significantly lower in the liver tissue obtained from the BDL rat model than in the normal liver. The CRP level was correlated with the expression of albumin in hepatocytes treated with LCA and siRNA-CRP. Tube formation was significantly decreased in HUVECs when they were treated with LCA or a combination of LCA and siRNA-CRP.

Conclusions: CRP seems to be involved in the abnormal formation of vessels in hepatic disease, and so it could be a useful diagnostic marker for hepatic disease. (Clin Mol Hepatol 2016;22:372-381)

Keywords: C-reactive protein; Bile duct ligation; Primary biliary cirrhosis; Hepatic disease

\section{INTRODUCTION}

The liver is the central organ for regulation of whole-body metabolism of carbohydrates, lipids and proteins as well as biotransformation and detoxification of endogenous metabolites. ${ }^{1}$ It is also the major site for biosynthesis of various serum constituents including albumin. ${ }^{2}$ In a normal liver, a steady-state hepatic vasculature with micro-vascularization allows the cells to perform elaborate enzymatic functions; however, altered microvasculature and metabolic gradient in the zonal organization of the liver in-

\section{Abbreviations:}

$B D L$, bile duct ligation; $C R P, C$-reactive protein; ECAR, extracellular acidification rate; HSC, hepatic stellate cell; HUVEC, human umbilical vein endothelial cell; H\&E; hematoxylin and eosin; IF, immunofluorescence; LCA, lithocholic acid; LDL, low density lipoprotein; NAFLD, nonalcoholic fatty liver disease; $\mathrm{OCR}$, oxygen consumption rate; $\mathrm{PBC}$, primary biliary cirrhosis; $\mathrm{TGF}$, transforming growth factor; $X F$, extracellular flux
}

\section{Corresponding author: Gi Jin Kim}

Department of Biomedical Science, CHA University, 59 Yatap-ro, Bundang-gu, Seongnam 13496, Korea

TelL +82-31-881-7145, Fax: +82-31-881-7249

E-mail: gjkim@cha.ac.kr 
duce a decrease in the sinusoid to hepatocyte ratio in damaged liver. ${ }^{3}$ These abnormal vessels caused by angiogenesis promote several types of hepatic failure such as hepatic fibrosis and cirrhosis. ${ }^{4}$ Although vascular structure is necessary for tissue growth and regeneration, accumulated injuries lead to pathologic situations in the liver, and contribute to the occurrence of inflammatory and chronic liver disease such as fibrosis and cirrhosis. ${ }^{5}$ Pathological angiogenesis is strongly correlated with progression of chronic liver disease and it acts as an initiator of fibrogenesis in liver tissues. Especially, cirrhosis of the liver induced by bile duct ligation (BDL) blocks the excretion of bile, and it is characterized by fibrosis and abnormal nodules via cholestatic liver injury. ${ }^{6}$ Bile duct ligation (BDL) and induces hepatic cirrhosis through cholestatic liver injury. These chronic cholestatic liver disease show high intracellular level of bile acids such as lithocholic acid (LCA), deoxycholic acid (DCA) and cholic acid (CDCA). Especially, LCA is a hydrophobic bile acid that is highly toxic. Scar formation and disorganization of the vessel structure in a cirrhotic liver can also aggravate local hypoxia. Hence, the formation of an irregular vasculature in the liver is related to the development of cirrhosis by enabling intrahepatic sinusoidal vessel remodeling and reducing effective hepatocyte perfusion. ${ }^{7}$

CRP (C-reactive protein) is an acute-phase protein indicating inflammation and it is secreted by the liver. It is principally synthesized and regulated in response to interleukin-6 (IL-6) or interleukin-1 $\beta$ (IL-1 $\beta$ ) in hepatocytes. ${ }^{8}$ It has been reported that CRP expression levels are associated with liver diseases such as hepatitis, nonalcoholic fatty liver disease (NAFLD), and fibrosis. CRP has also been considered to be a predictive factor for NAFLD, as its increased serum levels correlate with the level of insulin resistance. ${ }^{9,10}$ However, a possible role for CRP in abnormal vessel formation in hepatic diseases has not yet been reported. Hence, in the present study, we aimed to analyze the vessel structures in a rat liver fibrosis model induced by BDL, and to examine the expression of CRP in the rat model with BDL in order to determine whether there was a correlation between CRP levels and abnormal vascular formation. Finally, we evaluated the use of CRP as a diagnostic marker for liver diseases.

\section{MATERIALS AND METHODS}

\section{Animal models}

Seven-week-old male Sprague-Dawley rats (Orient Bio Inc.,
Seongnam, Korea) were housed in an air-conditioned animal facility under specific pathogen-free conditions. Liver cirrhosis was induced by common bile duct ligation as described previously. ${ }^{11}$ Briefly, rats were anesthetized with $75 \mathrm{mg} / \mathrm{mL}$ of Avertin (Sigma Aldrich, St. Louis, MO, USA) and their abdomens were disinfected and incised to expose the bile duct, which was then clamped with a suture in the upper and lower regions. The bile duct was then ligated between the ligatures. Following the procedure, the abdominal layers were closed and sutured separately. After 1, 2, 3 and 5 weeks, the rats were sacrificed and their livers were harvested. In all animal experimental processes, protocols approved by the Institutional Review Board (IRB) of CHA General Hospital, Seoul, Korea (IACUC-140009).

\section{Cell culture}

Rat hepatocytes (WB-F344) were cultured in $\alpha$-MEM (GIBCO $B R L$, Langley, OK, USA) medium, supplemented with $1 \%$ penicillin, streptomycin (Pen-Strep, GIBCO BRL), and 5\% fetal bovine serum (FBS, GIBCO BRL). Human umbilical vein endothelial cells (HUVECs) were cultured in EGM-2 medium at (GIBCO BRL) $37^{\circ} \mathrm{C}$, in a $5 \% \mathrm{CO}_{2}$ incubator. Additionally, cells were treated with siRNA-CRP and scrambled siRNA for 12 hours followed by treatment with $100 \mu \mathrm{M}$ lithocholic acid (LCA, Sigma Aldrich) for 12 hours.

\section{In vitro angiogenesis assay}

To confirm the effect of CRP on tube formation of HUVECs, the cells were stained with Alexa Fluor 488 AcLDL (Invitrogen Corporation, CA, USA). Then HUVECs $\left(5 \times 10^{4}\right.$ cells $\left./ \mathrm{mL}\right)$ were seeded on coverslips pre-coated with 5\% Matrigel (Sigma-Aldrich) and cultured with or without siRNA-CRP and LCA at $37^{\circ} \mathrm{C}$, in a $5 \% \mathrm{CO}_{2}$ incubator. The cultured cells were washed with cold phosphatebuffered saline (PBS). The branch lengths of the tube were measured and quantified using Image J program. All experiments were performed in triplicate.

\section{Quantitative real-time polymerase chain reaction (qRT-PCR)}

Total RNA was isolated from cells and liver tissues using the Trizol reagent (Invitrogen). cDNA synthesis was performed with $250 \mathrm{ng}$ of total RNA and Superscript III reverse transcriptase (Invitrogen). qRT-PCR was performed with SYBR EX taq (Roche, Branchburg, NJ, USA). The CDNA was amplified by PCR using the 
following conditions: 5 seconds at $95^{\circ} \mathrm{C}, 45$ cycles at $95^{\circ} \mathrm{C}$ for 30 seconds, $60^{\circ} \mathrm{C}$ for 15 minutes, $70^{\circ} \mathrm{C}$ for 15 minutes, and $72^{\circ} \mathrm{C}$ for 7 minutes. The sequences of the primers used were as follows: rat CRP, 5'-GCT TTT GGT CAT GAA GAC ATG TC-3' (Forward) and 5'TCA CAT CAG CGT GGG CAT AG-3' (Reverse). The primers for rat GAPDH were 5'-TCC CTC AAG ATT GTC AGC AA-3' (Forward) and 5'-AGA TCC ACA ACG GAT ACA TT-3' (Reverse). Each sample was analyzed in duplicate and expression of the CRP gene was normalized to that of GAPDH, the internal control.

\section{Western blot}

Cells and liver tissues were lysed in RIPA buffer containing protease inhibitor cocktail (Roche) and phosphatase inhibitor (SigmaAldrich). A total of 45 ug protein extracts were separated in 10\% sodium dodecyl sulfate polyacrylamide gels (SDS-PAGE). The separated proteins were transferred onto PVDF membranes (Bio-Rad, Hercules, CA, USA). The membrane was incubated with rabbit anti-CRP (1:1,000, Santa Cruz Biotechnology, Dallas, TX, USA), mouse anti-albumin (1:1,000 Santa Cruz), mouse anti-VEGF, rabbit anti-VEGFR1, mouse anti-endoglin (1:1,000 R\&D Systems, Abingdon, UK), and rabbit anti- $\beta$-actin (1:3,000, Sigma Aldrich) at $4^{\circ} \mathrm{C}$ overnight. The membrane was incubated with horseradish peroxidase (HRP)-conjugated secondary antibody (anti-rabbit IgG [1:20,000, Bio-Rad Laboratories, Hercules, CA, USA] or antimouse IgG [1:5,000, Santa Cruz]) for 1 hour at room temperature. The bands were detected using Clarity Western ECL kit (BioRad).

\section{Histological analysis}

Formalin-fixed liver tissues were embedded in paraffin and cut into $5 \mu \mathrm{m}$-thick sections and stained with the hematoxylin and eosin (H\&E) staining procedure. The portal vein diameter was assessed using Zeiss Axioskop2 MAT microscope (Carl Zeiss MicroImaging, Oberkochen, Germany) and quantified using Image J program. All experiments were performed in triplicate.

\section{Immunofluorescence staining}

To analyze the localization of CRP in the liver tissues, frozen liver sections were treated with the Blocking Solution (DAKO, Glostrup, Produktionsvej, Denmark) at room temperature for 40 minutes, and rabbit anti-CRP (1:50, Santa Cruz) was applied to the sections at $4^{\circ} \mathrm{C}$ overnight. Samples were washed with PBS, and incubated with Alexa Fluor 568 (1:100, Invitrogen)-conjugated secondary antibody at room temperature for 1 hour. To detect the co-localization of albumin and CRP in WB-F344 cells incubated with siRNA-CRP and LCA, WB-F344 cells were fixed with 100\% methanol (Merck, NJ, USA). The cells were reacted with Blocking Solution (Dako) for 1 hour at room temperature and mouse antialbumin (1:50, Santa Cruz) and rabbit anti-CRP (1:50, Santa Cruz) at $4^{\circ} \mathrm{C}$ overnight. After reaction, the cells were incubated with $\mathrm{Al}$ exa 488 and 568 (1:100, Invitrogen)-conjugated secondary antibody at room temperature for 1 hour. The slides were stained with 4', 6-diamidino-2-phenylindole (DAPI). The images were observed with a fluorescence microscope (Nikon, Tokyo, Minato, Japan). All experiments were performed in triplicate.

\section{Extracellular flux (XF) glycolysis and cell mitochondrial stress assay}

To analyze the effect of CRP on glycolysis ability or the level of mitochondrial stress in WB-F344 cells, oxygen consumption rate $(O C R)$ and extracellular acidification rate (ECAR) were investigated using XF-24 cell culture assay (Seahorse Bioscience, North Billerica, MA, USA) according to the manufacturer's procedure. Briefly, WB-F344 cells $\left(3 \times 10^{3}\right)$ were seeded and attached for 3 hours. Then, the culture medium was replaced and cells were treated with LCA and siRNA-CRP. After incubation, drug compounds related to glycolysis ability (Glucose: $10 \mathrm{mM}$, Oligomycin: $1.0 \mu \mathrm{M}$, 2-DG: $50 \mathrm{mM}$ ) and mitochondrial stress (Oligomycin: $1.0 \mu \mathrm{M}$, FCCP: $0.5 \mu \mathrm{M}$, Rotenone: $0.5 \mu \mathrm{M}$ ) were mixed and added to the $\mathrm{pH} 7.4$ culture medium. OCR and ECAR were analyzed using an XF analyzer (Seahorse Bioscience). All experiments were performed in triplicate.

\section{Statistical analysis}

All experiments were repeated twice, with data representing mean \pm standard error (S.E). The data was analyzed for statistical significance at a $P$-value $<0.05$ using Student's $t$-test.

\section{RESULTS}

\section{Analysis of vascular structure and secreted angiogenic factors in the rat model with BDL}

To analyze the vascular structure in liver tissues obtained from 


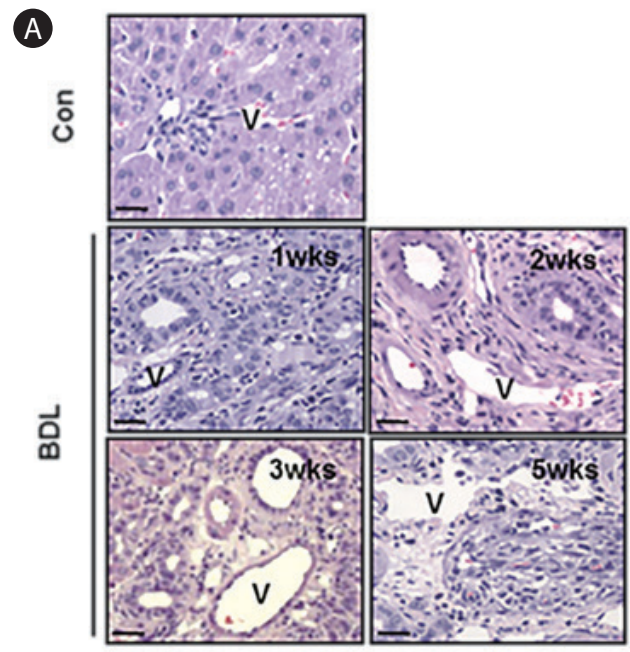

B
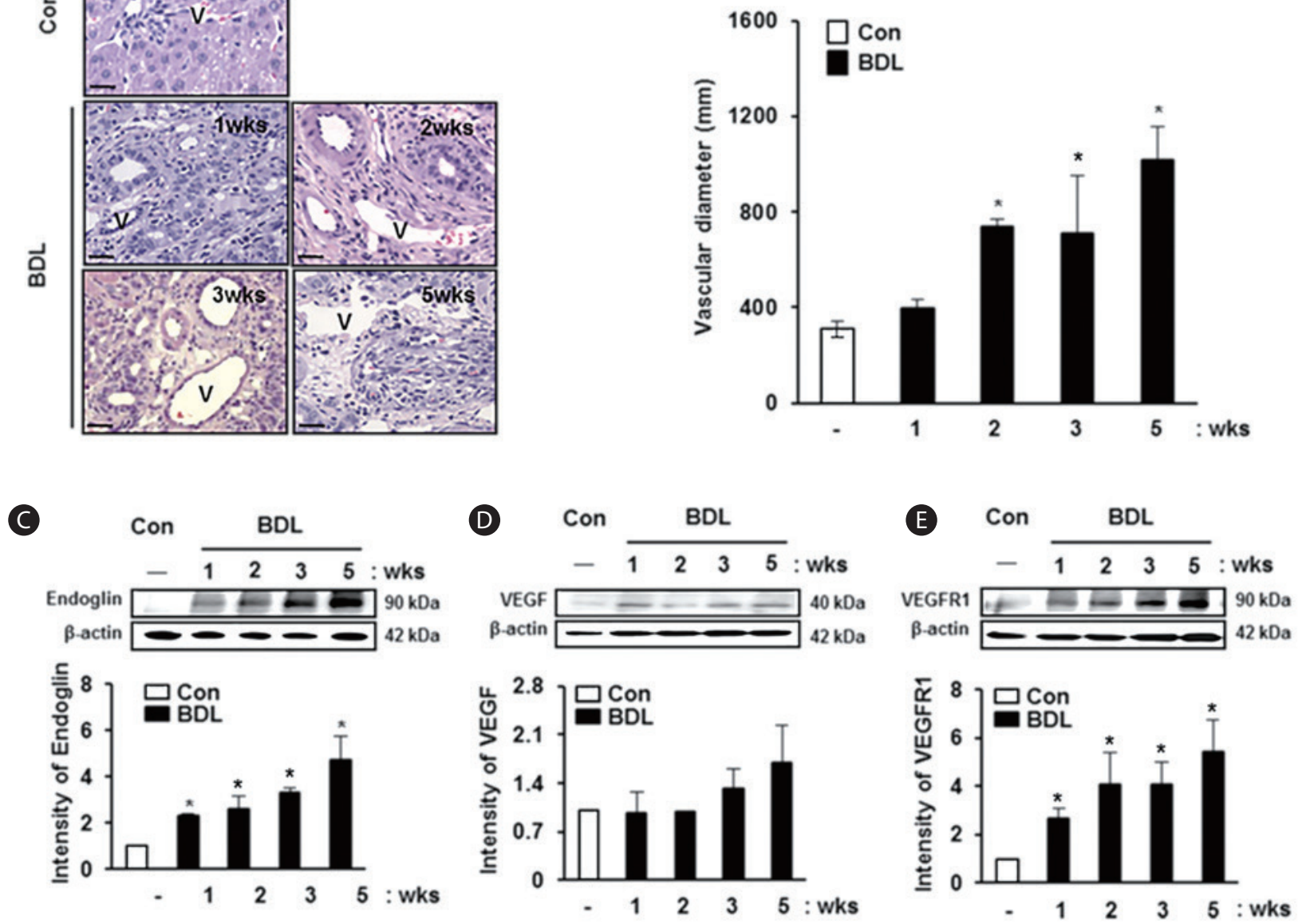

Figure 1. Vessel formation in the liver of the BDL rat model. (A) Histological analysis with H\&E staining (Original magnification, $\times 200)$. (B) Quantification of the portal vein diameter. Expression intensities of endoglin (C), VEGF (D), and VEGFR1 (E). $\beta$-actin was used as an internal control. All experiments were performed in duplicate. " $P<0.05$, Con versus others. Con, Sham control; BDL, bile duct ligation group; $\mathrm{V}$, portal vein.

the BDL model, the diameter of the portal vein was calculated. The diameter of the portal vein was increased depending on the degree of cirrhosis in the rat model with BDL compared with normal liver tissues (Fig. 1A). Especially, the diameter of the portal vein was significantly increased compared to that in a normal rat liver, and this finding was observed in tissues obtained from rats in the BDL group at 2 weeks and 5 weeks $(P<0.005$, Fig. 1B); however, statistical significance was not reached on analyzing tissues at 1 and 3 weeks. Also, we examined the expression of angiogenic factors in the BDL rat model. The expression of endoglin was increased in BDL rat liver tissues according to liver cirrhosis, and it showed a significant difference between the groups $(P<0.05$, Fig. 1C). The expression levels of vascular endothelial growth factor (VEGF) and vascular endothelial growth factor receptor 1 (VEGFR1) were increased with progression of cirrhosis in the BDL group compared with normal rat liver tissues, although statistical significance was not reached (Fig. 1D, E). These results suggest that the diameter of the portal vein in damaged liver tissues increases abnormally and the angiogenic factors are overexpressed with severity depending on the stage of progression of liver cirrhosis.

\section{Correlation between levels of CRP, albumin and various angiogenic factors in the rat BDL model}

To analyze the relationship between CRP and liver injury, we demonstrated its expression in liver tissues of the rat model with BDL. CRP mRNA expression was significantly decreased in liver tissues of the rat model with $B D L$ compared with normal rat liver tissues $(P<0.05$, Fig. $2 A)$. In addition, the expression of CRP at 

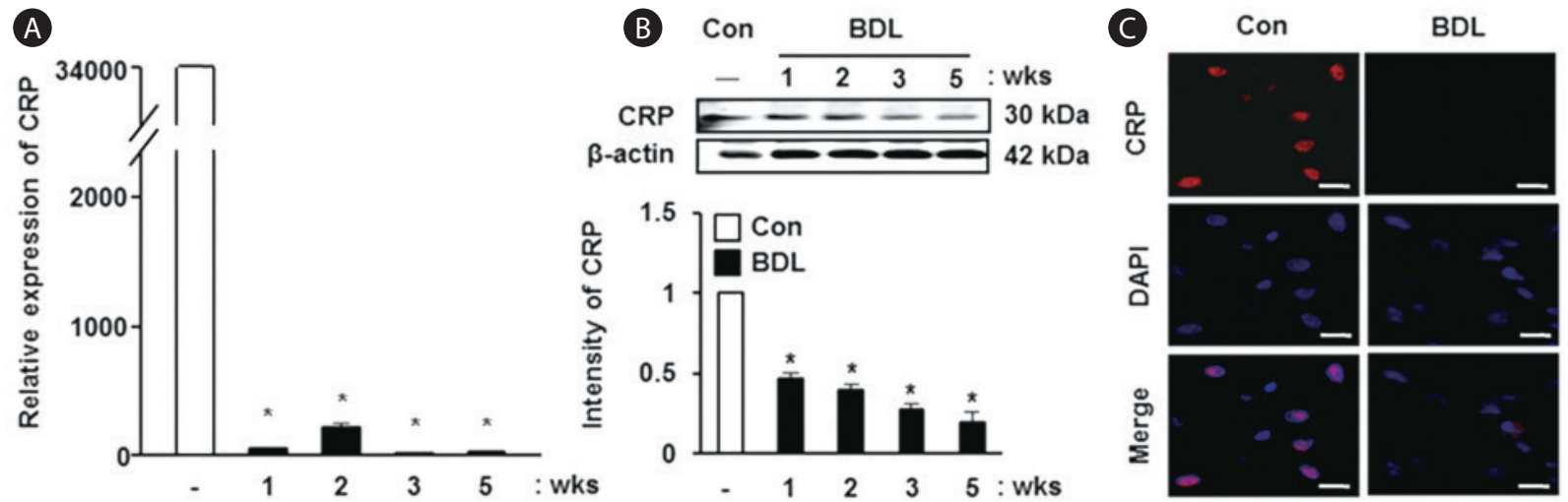

D
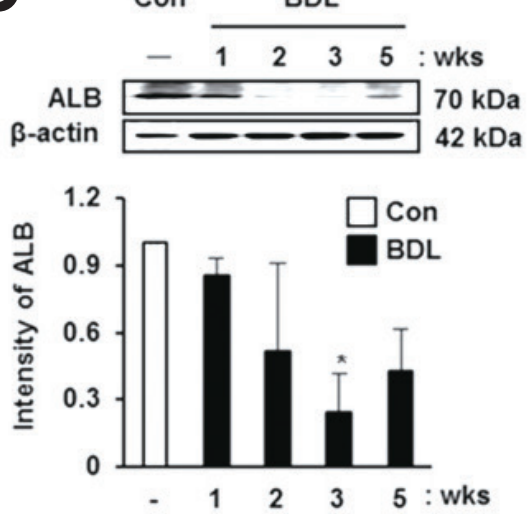

E

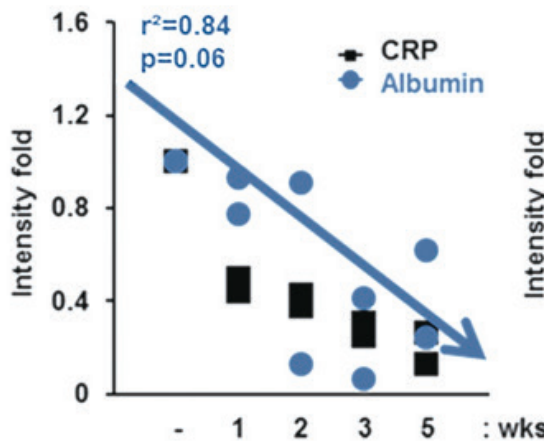

F

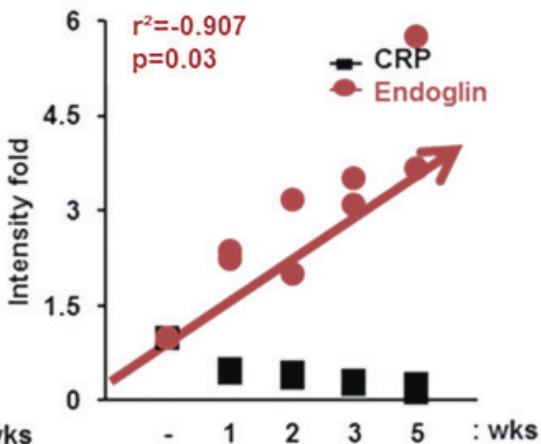

Figure 2. Correlation of CRP with albumin and endoglin in the BDL rat model. (A) Relative mRNA expression of CRP compared with GAPDH expression by real-time RT-PCR. (B) Western blotting analysis of CRP expression. $\beta$-actin was used as an internal control. All experiments were performed in duplicate. ${ }^{*}<<0.05$, control versus others. (C) Localization and expression of CRP by immunofluorescence analysis at 5 weeks. Original magnification, $\times 400$. (D) Expression of albumin by Western blotting. $\beta$-actin was used as an internal control. All experiments were performed in duplicate. " $P<0.05$, control versus others. (E) Correlation between CRP and albumin by Western blotting. (F) Correlation between CRP and endoglin by Western blotting. Con, Sham control; BDL, bile duct ligation group; ALB, albumin.

the protein level was significantly reduced in the BDL rat group depending on the progression of liver cirrhosis $(P<0.05$, Fig. 2B). The localization of CRP was observed in the nuclei of hepatocytes, and its expression was decreased in the liver tissue obtained from the rat model with BDL (Fig. 2C). Similar to the expression changes in CRP, the expression of albumin was also decreased in the rat model with BDL (Fig. 2D). Based on these findings, we analyzed the correlation between CRP and albumin, and a positive correlation was observed in spite of the $P$-value being $0.06\left(r^{2}=0.84\right.$, Fig. $\left.2 \mathrm{E}\right)$. However, the values for correlation between endoglin and CRP showed an inverse relationship $\left(r^{2}=-\right.$ $0.907, P=0.03$, Fig. 2F). These data show that the expression of CRP and albumin decreases with aggravation of liver cirrhosis and they show a positive correlation, whereas the relationship between CRP and endoglin, an angiogenic factor, displays an in- verse correlation.

\section{Effect of CRP on albumin expression in rat hepatocyte cells exposed to LCA or treated with siRNA CRP}

To analyze the effect of CRP on albumin expression in rat hepatocyte cells, we treated the cells with LCA or siRNA-CRP. The expression of CRP was the highest in the control group, whereas it was significantly decreased in the groups treated with LCA or SiRNA-CRP $(P<0.05$, Fig. 3A). Likewise, the expression of albumin was lower in the groups exposed to siRNA-CRP or LCA compared with the control group (Fig. 3B). The localization of CRP and albumin was noted in the cytoplasm of normal rat hepatocytes, but their expressions were reduced with LCA or siRNA-CRP treatment 

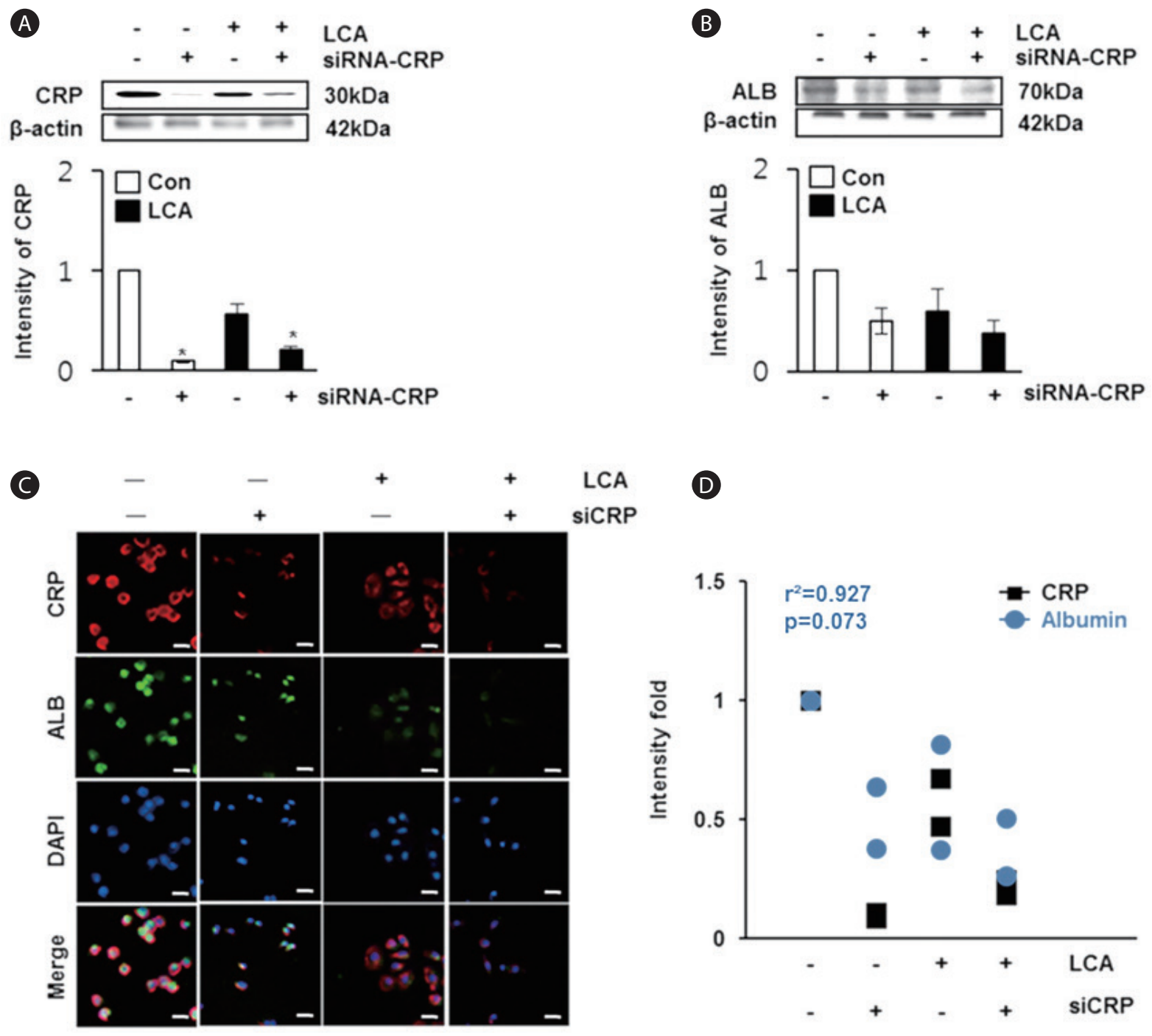

Figure 3. Correlation between CRP and ALB in WB-F344 cells with treated LCA and siRNA-CRP. Expression levels of (A) CRP and (B) albumin by Western blotting. $\beta$-actin was used as an internal control. All experiments were performed in duplicate. ${ }^{*} P<0.05$, control versus others. (C) Co-localization of $C R P$ and albumin by immunofluorescence analysis. Original magnification, $\times 200$. Con, normal WB-F344 cells; LCA, LCA-treated group; siCRP, siRNA-CRP treated group; ALB, albumin.

(Fig. 3C). On the basis of these results, we obtained a positive correlation coefficient of $r^{2}=0.927$ between CRP and albumin in rat hepatocytes (Fig. 3D). These findings suggest that their expressions are decreased in LCA and siRNA-CRP treated groups and it shows their parallel relevance, similar to the in vivo data.

\section{Effect of CRP levels on glycolysis and mitochondrial stress in rat hepatocytes}

Because assessing metabolic flux is essential to understanding cellular metabolism, glycolysis and mitochondria function in ad- herent cells are calculated by extracellular acidification rate (ECAR) and rate of mitochondrial oxygen consumption (OCR), respectively. To examine the effect of cellular metabolism by CRP levels in rat hepatocytes, we analyzed the changes in mitochondrial respiration by oxygen consumption and the changes in glycolysis by extracellular acidification rate using XF-24 cell culture assay. With respect to the extracellular flux changes in glycolysis (ECAR), it was highest in the control rat hepatocytes compared to the other groups, and it was significantly decreased in the groups treated with LCA or SIRNA-CRP and the combination treatment group had the lowest value for ECAR $(P<0.05$, Fig. $4 A)$. This indi- 
A

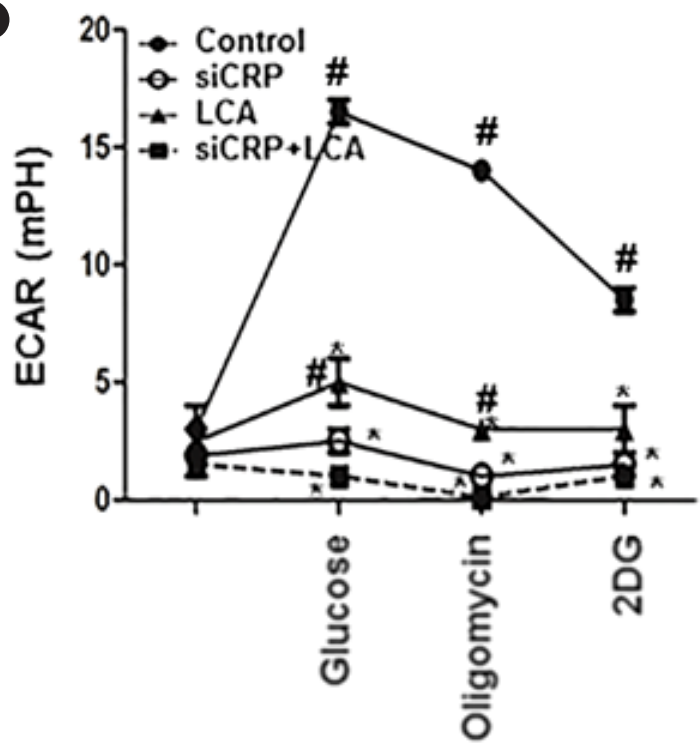

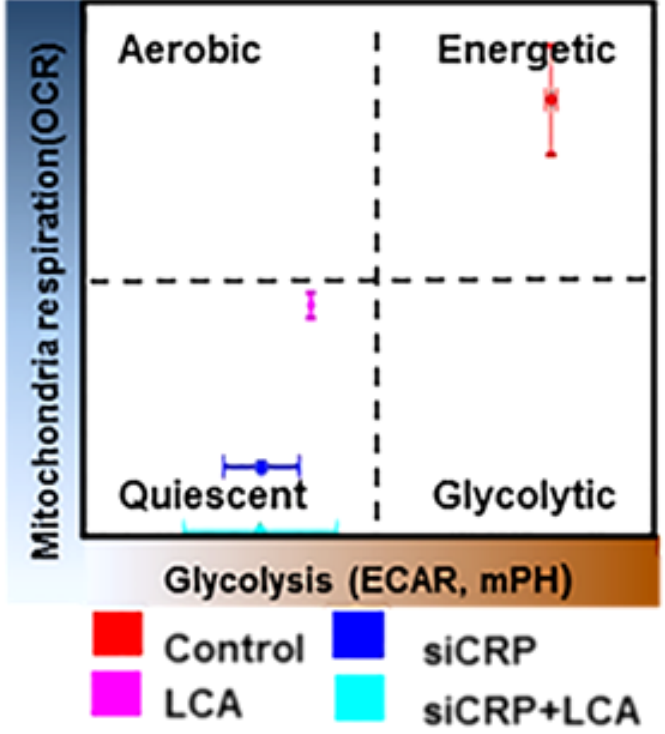

B
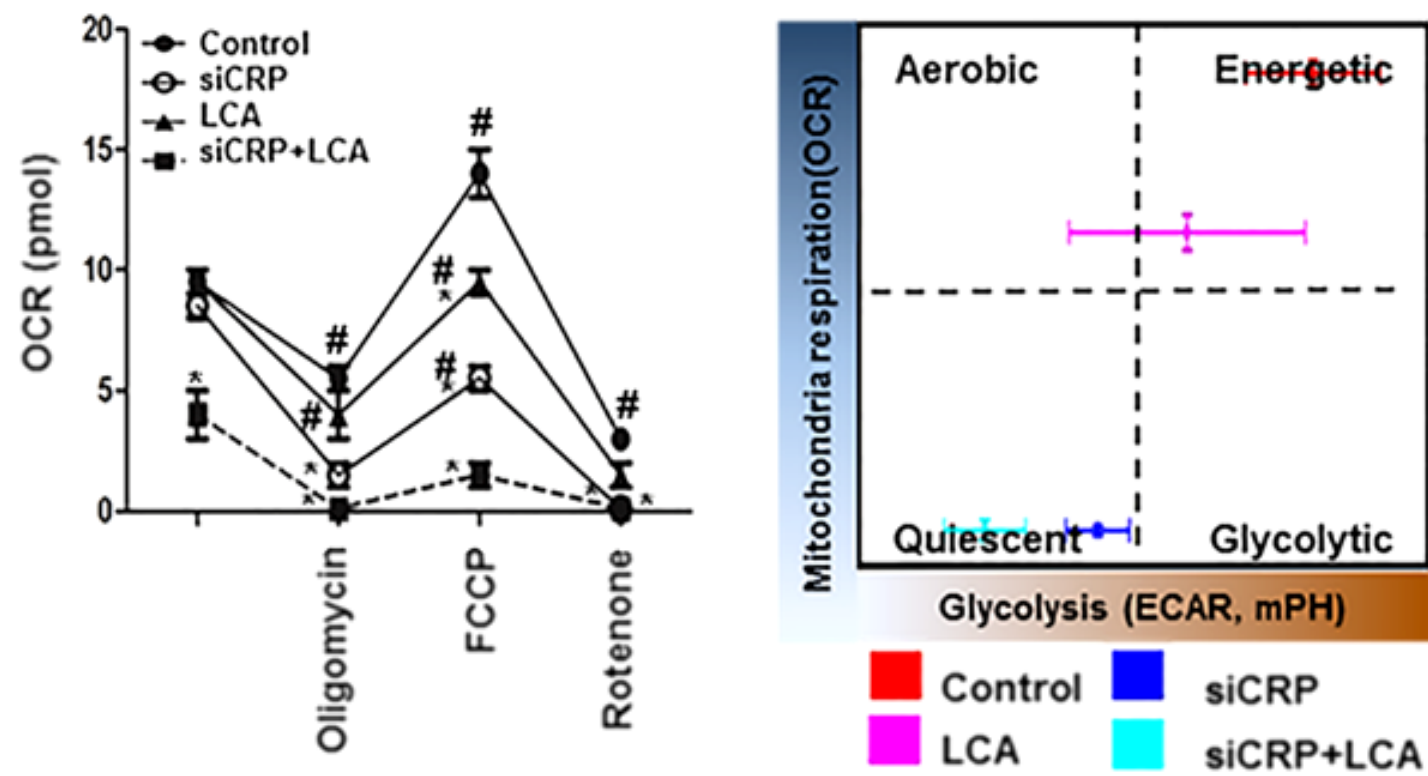

Figure 4. Glycolysis and mitochondrial stress. (A) Measurement of ECAR by the XF glycolysis stress test and the cell energy phenotype test. (B) Analysis of OCR by the XF cell mitochondrial stress test and the cell energy phenotype test. ${ }^{*} P<0.05$, control versus others; ${ }^{\sharp} P<0.05$, siRNA-CRP+LCA versus others. LCA, LCA-treated group; siCRP, siRNA-CRP treated group; XF; extracellular flux; ECAR, extracellular acidification rate; OCR, oxygen consumption rate.

cated that control rat hepatocytes were considered to be in an energetic state, whereas the hepatocytes in the groups treated with LCA or siRNA-CRP were more quiescent in terms of being in a certain energy consumption state. In the mitochondrial respiration assay, the OCR levels were significantly higher in the control group than in the groups treated with LCA or siRNA-CRP. Also, the OCR level was the lowest in the group treated with both LCA and siRNA-CRP $(P<0.05$, Fig. 4B). These findings suggest that LCA and siRNA-CRP treated rat hepatocytes became more metabolically quiescent compared with untreated hepatocytes. Therefore, CRP can play a role in regulating glycolysis and mitochondrial stress in hepatocytes by decreasing glycolysis but increasing mitochondrial stress. 
A
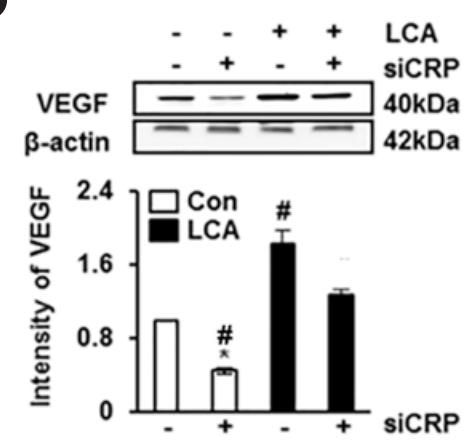

B
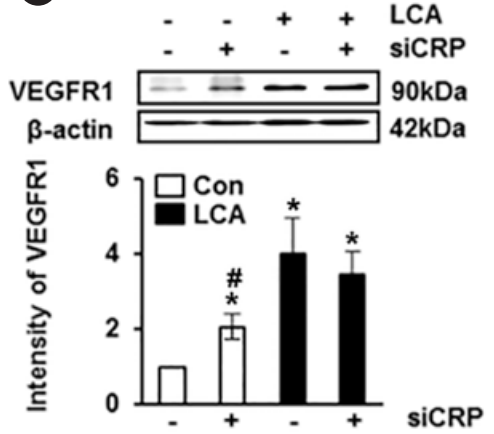

C
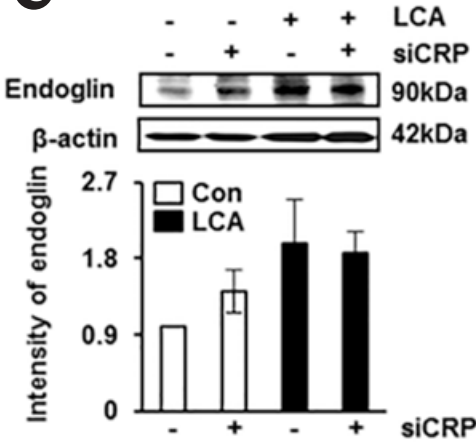

(D)

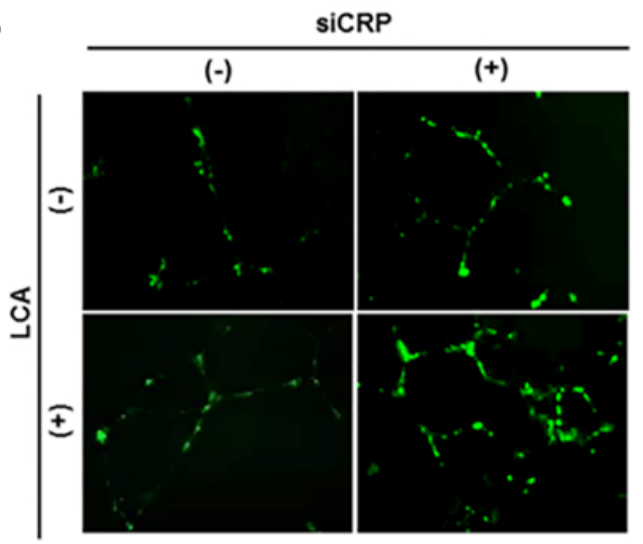

E

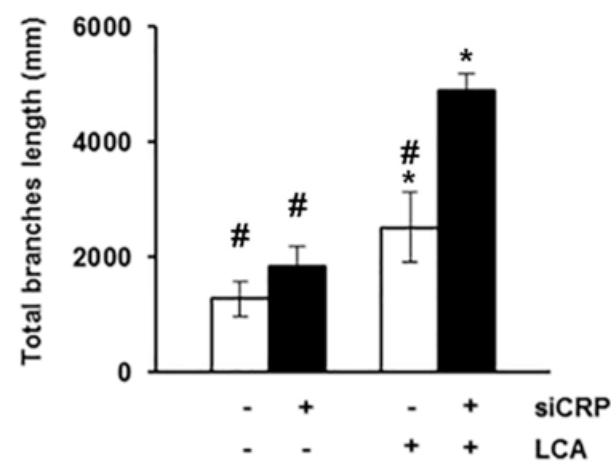

Figure 5. Expression of angiogenic markers in WB-F344 cells and tube formation in HUVECs treated with LCA and siRNA-CRP. (A) Comparison of VEGF and $\beta$-actin expression levels by Western blotting. VEGFR1 (B) and endoglin (C) expression levels. (D) Analysis of tube formation stained with low-density lipoprotein in HUVECs treated with LCA and siRNA-CRP. Original magnification, $\times 200$. (E) Quantification of the total length of branches in HUVECs treated with LCA and siRNA-CRP. " $P<0.05$, control versus others; ${ }^{\#} P<0.05$, siRNA-CRP+LCA versus others. Con, normal WB-F344 cells; LCA, LCA-treated group; siCRP, siRNA-CRP treated group.

\section{Effect of CRP levels on the expression of angiogenic factors and vessel formation}

To investigate the effect of CRP on expressions of angiogenic factors in rat hepatocytes, we analyzed the expression of VEGF, VEGFR1, and endoglin. The expression of VEGF was significantly increased in rat hepatocytes exposed to LCA than in the untreated control group; otherwise, its expression was significantly decreased in the group treated with siRNA-CRP than in control $(P<0.05$, Fig. 5A). The expressions of VEGFR1 and endoglin were also higher in the LCA-treated groups than in the untreated group; however, their expressions were decreased by siRNA-CRP treatment although the difference was not statistically significant (Fig. 5B, C). Next, we demonstrated the effect of CRP on vascular formation in HUVECs according to treatment with LCA or siRNACRP. Vascular formation was increased in the LCA-treated groups, and the group treated with both LCA and siRNA-CRP showed the largest extent of vascular formation (Fig. 5D). Especially, the quantified total length of vessels was statistically increased in the LCA and siRNA-CRP combination treatment group compared with the untreated group $(P<0.05$, Fig. 5E). These findings indicate that the expression of angiogenic factors in rat hepatocytes and vascular formation in HUVECs are increased when these cells are co-treated with LCA and siRNA-CRP.

\section{DISCUSSION}

In the liver, the endothelium is a critical regulator of liver damage as vessels consisting of endothelial cells play a role in maintaining vascular homeostasis or they are involved in response to inflammation. ${ }^{12}$ In a rat model of liver cirrhosis, there is increased 
angiogenesis and proliferation of the bile duct which allows for higher ischemic tolerance. ${ }^{13}$ Particularly, the expression of angiogenic factors such as VEGF-A, B, and C or their receptors was extensively higher in chronic hepatitis and primary biliary cirrhosis (PBC) than in normal human liver tissues. ${ }^{14}$ The up-regulated angiogenic factors lead to the formation of abnormal vessels through defenestration and capillarization of the endothelium, which is considered to be the principal facilitator of hepatic injury in cirrhosis, thus increasing vascular resistance and leading to complications including portal hypertension. ${ }^{15,16}$ But to date, the cellular and molecular mechanisms causing liver cirrhosis due to endothelial angiogenesis are not fully understood.

Recently, it has been reported that increased CRP levels as part of the inflammatory response lead to endothelial dysfunction by reducing endothelial nitric oxide synthase and down-regulating angiogenesis as well as by an inverse correlation with functions of endothelial progenitor cells that participate in vascularization and secrete angiogenic growth factors. ${ }^{17,18}$ Also, Enjoji et al. reported that CRP and VEGF showed a positive correlation in acute cholangitis patients, but in chronic PBC patients, the correlation was not significant. ${ }^{19}$

In the present study, we found that abnormal vessel formation as well as expression of angiogenic factors was increased while the expression of CRP was decreased with progression of liver cirrhosis induced by bile duct ligation. This suggested that CRP might regulate abnormal vessel formation in liver cirrhosis. However, the correlations between levels of CRP and VEGF or VEGFR1 in a rat model with BDL were not significant, however, the correlation between levels of CRP and endoglin was significant $\left(r^{2}=-\right.$ $0.907, P=0.03)$. The reason for this occurrence may be that damage to hepatic cells including endothelial cells induced by liver cirrhosis also reduces the expression of VEGF and VEGFR1.

Generally, bile duct ligation (BDL) blocks the excretion of bile and leads to a build up of bile acids and induces hepatic cirrhosis as well as hypertension. ${ }^{20}$ Hence, the accumulation of LCA, a toxic secondary bile acid, induces destructive cholangitis as well as progression of bile duct obstruction by lipid dysbolism. ${ }^{21}$ Recently, it was shown that mitochondrial oxidative stress in hepatocytes is associated with changes in lipid metabolism as $\beta$-oxidation of overabundant free fatty acids is processed in mitochondria of the hepatocytes and it produces excessive reactive oxygen species (ROS). ${ }^{22}$ Furthermore, Meshkani et al. reported that CRP levels correlated with levels of lipid metabolism and insulin resistance was related to glucose metabolism, pointing to the possibility of CRP being involved in metabolism in the liver. ${ }^{23}$ However, our data shows that increased CRP was correlated with improved glucose metabolism in rat hepatocytes by extracellular flow (XF) analysis. This discrepancy may be due to the difference in cell types studied, concentrations of CRP applied or used, and the differences in the expression of glucose transporters such as GLUT2 and GLUT4 for various systems. ${ }^{24}$ Based on our analysis, we suggest that CRP can be involved in the metabolism in the liver in terms of glycolysis and mitochondrial respiration in hepatocytes during cirrhosis induced by bile duct ligation. Although CRP produced by hepatocytes has a positive correlation with albumin, known as a hepatic regeneration marker, ${ }_{1}^{25}$ CRP has been regarded as a possible biomarker for predicting several hepatic diseases including cirrhosis and morbidity of liver transplant patients. ${ }^{26}$ However, CRP was not proved to be a diagnostic marker for severe end-stage liver cirrhosis. Therefore, this is the first report which shows the usefulness of CRP as a prognostic marker in chronic liver cirrhosis in a rat model with BDL. As the expression of CRP decreased depending on the progression of chronic liver cirrhosis, it correlated with albumin levels $\left(r^{2}=0.84\right.$ and $r^{2}=0.927$ in the rat model with BDL and hepatocytes in culture, respectively). The reduced levels of CRP also correlated with reduced levels of angiogenic factors leading to reduced abnormal vascular formation.

In conclusion, the formation of abnormal vessel structure was increased in our BDL rat liver model with aggravation of liver cirrhosis, whereas the expression of CRP was decreased. This means that there is an inverse correlation between CRP expression and abnormal vascular formation. Based on these findings, we suggest that CRP can be used as a diagnostic marker for abnormal vessel formation in chronic liver disease.

\section{Funding support}

This work was supported by a grant from Korea Food \& Drug Administration in 2016 (14172MFDS974).

\section{Conflicts of Interest}

The authors have no conflicts to disclose.

\section{REFERENCES}

1. Bode JG, Albrecht U, Häussinger D, Heinrich PC, Schaper F. Hepatic acute phase proteins--regulation by IL-6- and IL-1-type cytokines involving STAT3 and its crosstalk with NF-kappaB-dependent signaling. Eur J Cell Biol 2012;91:496-505.

2. Kwong E, Li Y, Hylemon PB, Zhou H. Bile acids and sphingosine1-phosphate receptor 2 in hepatic lipid metabolism. Acta Pharm Sin 
Ji Hye Jun, et al.

Effect of CRP on vascular structure of hepatic disease

B 2015;5:151-157.

3. Gaudio E, Pannarale L, Franchitto A, Onori P, Marinozzi G. Hepatic microcirculation as a morpho-functional basis for the metabolic zonation in normal and pathological rat liver. Ital J Anat Embryol 1995;100(Suppl 1):419-428.

4. Furutani Y, Shiozaki-Sato Y, Hara M, Sato Y, Kojima S. Hepatic fibrosis and angiogenesis after bile duct ligation are endogenously expressed vasohibin-1 independent. Biochem Biophys Res Commun 2015;463:384-388.

5. Elpek GO. Angiogenesis and liver fibrosis. World J Hepatol 2015;7:377-391.

6. Vanheule E, Geerts AM, Van Huysse J, Schelfhout D, Praet M, Van Vlierberghe $H$, et al. An intravital microscopic study of the hepatic microcirculation in cirrhotic mice models: relationship between fibrosis and angiogenesis. Int J Exp Pathol 2008;89:419-432.

7. Fernandez M, Semela D, Bruix J, Colle I, Pinzani M, Bosch J. Angiogenesis in liver disease. J Hepatol 2009;50:604-620.

8. Zhao J, Liu J, Pang X, Wang S, Wu D, Zhang X, et al. Angiotensin II induces C-reactive protein expression via AT1-ROS-MAPK-NF-kappaB signal pathway in hepatocytes. Cell Physiol Biochem 2013;32:569-580.

9. Riquelme A, Arrese M, Soza A, Morales A, Baudrand R, Pérez-Ayuso $R M$, et al. Non-alcoholic fatty liver disease and its association with obesity, insulin resistance and increased serum levels of C-reactive protein in Hispanics. Liver Int 2009;29:82-88.

10. Chunming L, Jianhui S, Hongguang Z, Chunwu Q, Xiaoyun H, Lijun Y, et al. The development of a clinical score for the prediction of nonalcoholic steatohepatitis in patients with nonalcoholic fatty liver disease using routine parameters. Turk J Gastroenterol 2015;26:408-416.

11. Mahmoud MF, Swefy SE, Hasan RA, Ibrahim A. Role of cannabinoid receptors in hepatic fibrosis and apoptosis associated with bile duct ligation in rats. Eur J Pharmacol 2014;742:118-124.

12. Gupta TK, Toruner M, Chung MK, Groszmann RJ. Endothelial dysfunction and decreased production of nitric oxide in the intrahepatic microcirculation of cirrhotic rats. Hepatology 1998;28:926-931.

13. Raftery MJ, Seron D, Hartley B, Koffman G, Cameron JS. Immunohistological analysis of the early renal allograft biopsy. Transplant Proc 1989;21:280-281.

14. Franchitto A, Onori P, Renzi A, Carpino G, Mancinelli R, Alvaro D, et al. Expression of vascular endothelial growth factors and their receptors by hepatic progenitor cells in human liver diseases. Hepa- tobiliary Surg Nutr 2013;2:68-77.

15. Iwakiri Y, Groszmann RJ. Vascular endothelial dysfunction in cirrhosis. J Hepatol 2007;46:927-934.

16. Iwakiri Y. Endothelial dysfunction in the regulation of cirrhosis and portal hypertension. Liver Int 2012;32:199-213.

17. Fasing KA, Nissan BJ, Greiner JJ, Stauffer BL, DeSouza CA. Influence of elevated levels of C-reactive protein on circulating endothelial progenitor cell function. Clin Transl Sci 2014;7:137-140.

18. Suh W, Kim KL, Choi JH, Lee YS, Lee JY, Kim JM, et al. C-reactive protein impairs angiogenic functions and decreases the secretion of arteriogenic chemo-cytokines in human endothelial progenitor cells. Biochem Biophys Res Commun 2004;321:65-71.

19. Enjoji M, Nakamuta M, Yamaguchi K, Ohta S, Kotoh K, Fukushima $M$, et al. Clinical significance of serum levels of vascular endothelial growth factor and its receptor in biliary disease and carcinoma. World J Gastroenterol 2005;11:1167-1171.

20. Ackermann D, Vogt B, Escher G, Dick B, Reichen J, Frey BM, et al. Inhibition of 11beta-hydroxysteroid dehydrogenase by bile acids in rats with cirrhosis. Hepatology 1999;30:623-629.

21. Chen $P$, Zeng $H$, Wang $Y$, Fan $X, X u C$, Deng $R$, et al. Low dose of oleanolic acid protects against lithocholic acid-induced cholestasis in mice: potential involvement of nuclear factor-E2-related factor 2-mediated upregulation of multidrug resistance-associated proteins. Drug Metab Dispos 2014;42:844-852.

22. Liu W, Baker RD, Bhatia T, Zhu L, Baker SS. Pathogenesis of nonalcoholic steatohepatitis. Cell Mol Life Sci 2016;73:1969-1987.

23. Meshkani R, Adeli K. Hepatic insulin resistance, metabolic syndrome and cardiovascular disease. Clin Biochem 2009;42:1331-1346.

24. Xi L, Xiao C, Bandsma RH, Naples M, Adeli K, Lewis GF. C-reactive protein impairs hepatic insulin sensitivity and insulin signaling in rats: role of mitogen-activated protein kinases. Hepatology 2011;53:127-135

25. Park WB, Lee KD, Lee CS, Jang HC, Kim HB, Lee HS, et al. Production of C-reactive protein in Escherichia coli-infected patients with liver dysfunction due to liver cirrhosis. Diagn Microbiol Infect Dis 2005;51:227-230.

26. Di Martino V, Coutris C, Cervoni JP, Dritsas S, Weil D, Richou C, et al. Prognostic value of $\mathrm{C}$-reactive protein levels in patients with cirrhosis. Liver Transpl 2015;21:753-760. 